\title{
Generalized hydrodynamics and extended irreversible thermodynamics
}

\author{
D. Jou and C. Pérez-García \\ Departament de Termologia, Universitat Autónoma de Barcelona, Bellaterra, Catalonia, Spain \\ L. S. García-Colín, ${ }^{*}$ M. López de Haro, and R. F. Rodríguez \\ Instituto de Investigaciones en Materiales, Universidad Nacional Autónoma de México, \\ Apartado Postal 70-360, 04510 México, Distrito Federal, Mexico \\ (Received 16 July 1984; revised manuscript received 26 October 1984)
}

\begin{abstract}
The thermodynamic implications of the first deviations with respect to the classical hydrodynamic behavior in high-frequency, short-wavelength phenomena are examined. The constitutive equations arising from an extended irreversible-thermodynamic formalism taking into account spatial inhomogeneities in the space of state variables are compared with those used in generalized hydrodynamics. The so-called exponential model for the memory function of the transverse-velocity correlation function is derived under the assumptions of extended irreversible thermodynamics only. Furthermore, it is also shown how more complicated memory functions can be derived. The results are carefully analyzed and compared with some microscopic derivations.
\end{abstract}

\section{INTRODUCTION}

The classical theory of linear irreversible thermoydnamics (LIT) provides a thermodynamic framework to the classical linear constitutive equations of hydrodynamics, namely, the Fourier and Newton-Stokes laws. The ability to perform experiments (light and neutron scattering) or computer simulations in hydrodynamic systems in a high-frequency, short-wavelength regime has motivated a very strong theoretical interest in providing a theoretical framework for the description of such experiments. This theoretical interest has been focused on the generalization of constitutive equations to the high-frequency, shortwavelength regime. As we have pointed out recently, ${ }^{1}$ in contrast with the classical linear situation, such an effort has no thermodynamic counterpart, because the classical LIT is only valid in the limit of very short frequencies and very long wavelengths. The aim of this paper is to point out a formalism able to explore the thermodynamic implications and consequences of the first deviations of the constitutive equations with respect to the classical ones at high frequencies and moderately long wavelengths and to predict some restrictions which are in fact observed in known experimental situations. Furthermore, this formalism is capable of furnishing a thermodynamic interpretation of what is presently referred to as generalized hydrodynamics in at least a certain approximation.

This thermodynamic complement to the generalized constitutive laws is not restricted to linear problems, but is able to deal with nonlinear ones. It provides an extension of LIT and gives an insight to its limits of validity and possible generalizations. Furthermore, it covers some range of an unexplored gap which in our opinion is of interest, because it provides a macroscopic, i.e., modelindependent, understanding of some physical phenomena, which often are analyzed by means of microscopic models or of ad hoc mathematical assumptions.

\section{EXTENDED IRREVERSIBLE THERMODYNAMICS}

The starting point of the extended irreversible thermodynamics (EIT) is the assumption that a regular and continuous function $\eta$ exists, playing the role of a generalized entropy and which depends on a specific set of nonconserved or fast variables, in addition to the ordinary conserved densities. In the case of an incompressible, insulating simple fluid we shall assume that the set of nonconserved quantities are the stress tensor $\mathscr{L}$ and an unspecified third-order tensor which shall be denoted by $\underline{\mathscr{P}}$ :

$$
\eta=\eta(e, \underline{\mathscr{P}}, \underline{\mathscr{P}}) \text {. }
$$

The rationale behind the motivation for including the tensor $\underline{\mathscr{P}}$ in Eq. (2.1) will become clear in a moment. It will suffice here to say that in this way spatial inhomogeneities in the space of state variables are included.

The extension of the local equilibrium assumption arising from Eq. (2.1) to lowest order in the nonconserved variables is given by ${ }^{2,3}$

$$
T \frac{d \eta}{d t}=\frac{d e}{d t}+v \alpha \stackrel{\mathscr{S}}{\frac{d \mathscr{S}}{d t}}+v \gamma \underline{\mathscr{P}}: \frac{d \mathscr{P}}{d t},
$$

where $\alpha$ and $\gamma$ are coefficients that depend on $v$ and $e$ only, and the double and triple dots in the second and third terms indicate double and triple contraction of the corresponding tensors, respectively. Here, $v=\rho^{-1}$.

The aim of EIT is to now provide a complete set of differential equations which govern the evolution in time of the new variables appearing in Eq. (2.1) in terms of a set of coefficients (equations of state), which may be determined from experiment or from a microscopic model. Thus we have now to supplement the energy conservation equation

$$
\rho \frac{d e}{d t}=-\stackrel{\mathscr{L}}{:(\operatorname{grad} \mathbf{u})^{s}}
$$


$\left({ }^{\circ}\right)^{s}$ denoting the symmetric traceless part of the corresponding quantity and $\mathbf{u}$ the hydrodynamic velocity, with equations for the other variables.

For this purpose we introduce a second assumption, namely, that $\eta$ satisfies a balance-type equation

$$
\rho \frac{d \eta}{d t}+\operatorname{div} \mathbf{J}_{\eta}=\sigma
$$

where $\mathbf{J}_{\eta}$ is the most general vector which may be constructed in the space of state variables and $\sigma$ is an undetermined scalar. When the subspace spanned by the nonconserved variables reduces to the void space, $\eta \rightarrow s$, the ordinary specific entropy, and both $\mathbf{J}_{\eta}$ and $\sigma$ must reduce to the entropy flux and entropy production, respectively, of linear irreversible thermodynamics. And it is only under such conditions that one can strictly require that $\sigma \geq 0$.

Accordingly, we may now write for $\mathbf{J}_{\eta}$ the expression

$$
\mathbf{J}_{\eta}=\beta \mathscr{\mathscr { S }}: \underline{\mathscr{P}} \text {, }
$$

where $\beta$ is a function of all scalar invariants in the space of state variables. However, since we shall be interested here in a very specific system, we shall approximate these equations regarding them as functions of $e$ and $v$ only. Computing the divergence of this quantity and making use of Eqs. (2.2) and (2.4), one readily arrives at the following expression for $\sigma$, namely,

$$
\begin{aligned}
\sigma= & -\frac{1}{T} \mathscr{\mathscr { L }}:(\operatorname{grad} \mathbf{u})^{s}+\frac{\alpha}{T} \mathscr{\mathscr { S }}: \frac{d \mathscr{\mathscr { S }}}{d t}+\frac{\gamma}{T} \underline{\mathscr{P}}: \frac{d \mathscr{P}}{d t} \\
& +\beta \mathscr{\mathscr { S }}: \operatorname{div} \underline{\mathscr{P}}+\beta \underline{\mathscr{P}}: \operatorname{grad} \mathscr{\mathscr { P }} .
\end{aligned}
$$

Furthermore, $\sigma$ may be computed in an entirely independent way by noticing that, being a scalar, it has to be the most general scalar that one can construct in the space of variables. Thus

$$
\sigma=\underline{X}: \underline{\mathscr{P}}+\underline{\mathbf{Y}}: \underline{\mathscr{P}},
$$

where $\underline{X}$ and $\underline{Y}$ are the most general tensors of order two and three, respectively, which can be constructed with $\mathscr{\mathscr { S }}$ and $\underline{\mathscr{P}}$. Hence

$$
\begin{aligned}
& \underline{\dot{X}}=\mu_{10} \underline{\mathscr{P}}+\mu_{11}(\underline{\mathscr{S}} \cdot \underline{\mathscr{S}})^{s}+\mu_{12}(\underline{\mathscr{P}}: \underline{\mathscr{P}})^{s}, \\
& \underline{\mathbf{Y}}=\mu_{20} \underline{\mathscr{P}} .
\end{aligned}
$$

Here, all the $\mu$ 's are, in principle, functions of all scalar invariants in the space of state variables, but consistent with the assumption introduced in Eq. (2.5), we shall take them to be functions of $e$ only. In this sense, $\beta$ and the $\mu$ 's are to be regarded as local equations of state.

Substitution of Eqs. (2.8) into Eq. (2.7) and comparison of the resulting equation with Eq. (2.6) leads to the desired result, namely, the time evolution equations for the nonconserved quantities. Indeed, keeping the lowestorder terms in $\underline{X}$ and $\underline{\mathscr{P}}$ we get that

$$
\begin{aligned}
\frac{d \mathscr{\mathscr { S }}}{d t} & =\frac{T}{\alpha}\left[\frac{1}{T}(\operatorname{grad} \mathbf{u})^{s}+\mu_{10} \stackrel{\mathscr{S}}{-}-\beta \operatorname{div} \underline{\mathscr{P}}\right], \\
\frac{d \underline{\mathscr{P}}}{d t} & =\frac{T}{\gamma}\left[-\beta \operatorname{grad} \underline{\mathscr{P}}+\mu_{20} \underline{\mathscr{P}}\right] .
\end{aligned}
$$

This set, together with Eq. (2.3), provides a complete set of equations for our chosen state variables that may be solved, in principle, provided that the coefficients $\alpha, \beta, \gamma$, $\mu_{10}$, and $\mu_{20}$ are known. By defining the relaxation times of $\mathscr{\mathscr { S }}$ and $\underline{\mathscr{P}}$, respectively, as

$$
\tau_{1}^{-1}=-\frac{\mu_{10} T}{\alpha}, \tau_{2}^{-1}=-\frac{T \beta}{\gamma},
$$

the set of equations (2.9) may be conveniently rewritten in the form of

$$
\begin{aligned}
& -\tau_{1} \frac{d \mathscr{\mathscr { S }}}{d t}=\frac{1}{\mu_{10} T}(\operatorname{grad} \mathbf{u})^{s}+\stackrel{\mathscr{S}}{ }-\gamma_{1} \operatorname{div} \underline{\mathscr{P}}, \\
& -\tau_{2} \frac{d \underline{\mathscr{P}}}{d t}=-\gamma_{2} \operatorname{grad} \underline{\mathscr{S}}+\underline{\mathscr{P}},
\end{aligned}
$$

where $\gamma_{1}=\beta / \mu_{10}$ and $\gamma_{2}=\beta / \mu_{20}$. Furthermore, when $\mathscr{P}=0$ and $\tau_{1}=0$ one must recover the well-known constitutive equations of ordinary hydrodynamics, which implies that $\left(\mu_{10} T\right)^{-1}=2 \eta_{s}$, where $\eta_{s}$ is the shear viscosity. This condition determines the coefficient $\mu_{10}$. Notice that within the approximation here considered, $\sigma \geq 0$ implies that both $\mu_{10}$ and $\mu_{20}$ have to be non-negative.

Emphasis should be placed on the fact that if $\tau_{2}$, which is in general smaller than $\tau_{1}$, is set equal to zero, so that $\gamma_{2} \underline{\mathscr{P}}=\operatorname{grad} \mathscr{\mathscr { S }}$, Eq. (2.11a) reduces to

$$
-\tau_{1} \frac{d \mathscr{\mathscr { S }}}{d t}=2 \eta_{s}(\operatorname{grad} \mathbf{u})^{s}+\mathscr{\mathscr { S }}-l^{2} \nabla^{2} \mathscr{\mathscr { S }},
$$

where $l^{2}=\gamma_{1} \gamma_{2}=2 T \eta_{s} \beta^{2} / \mu_{20}$ plays the role of a length describing the range of the spatial inhomogeneities in the variable $\mathscr{\mathscr { L }}$ and is a non-negative quantity. ${ }^{4}$ Equation (2.12) has, as we shall show in Sec. III, a strong bearing on the conceptualization of generalized hydrodynamics. It has also been used to discuss ultrasonic absorption in metals ${ }^{5}$ and some aspects of phonon hydrodynamics. ${ }^{6}$ Similarly, its analog for the case of a rigid heat conductor can be applied to the study of ultrafast thermometry. ${ }^{7}$

It is also important to stress that Eqs. (2.11) are a consequence of the structure proposed for the space of state variables of the system and the two postulates of extended irreversible thermodynamics only. In this sense, they are unquestionable mathematical objects. However, if a physical interpretation is sought for $\mathscr{P}$, then one has to resort either to experiment or to a microscopic theory. In the second case, a moment analysis of the singleparticle distribution function for a dilute gas obeying the Boltzmann equation shows that $\mathscr{P}$ plays the role of the "current" associated to the quantity $\mathscr{\mathscr { S }}^{8}$. It is clear from previous considerations ${ }^{9}$ that this variable is entirely superfluous in the case of gases, but nevertheless it has been found useful to generate a continuous-fraction expansion for the transport coefficients. ${ }^{10}$ This point will not be pursued here.

\section{EIT AND GENERALIZED HYDRODYNAMICS}

We shall now proceed to calculate the time correlation function of transverse-velocity fluctuations around equilibrium from the relaxation equations derived in Sec. II. It is important to point out that although this correlation 
function cannot be obtained from experiments, its calculation and analysis is worth pursuing since, on the one hand, it has been extensively studied by computer simulations ${ }^{11}$ and, on the other hand, it plays an essential role in establishing the connection between EIT and generalized hydrodynamics.

The transverse-velocity correlation function is defined in general as

$$
C\left(\mathbf{r}, t ; \mathbf{r}^{\prime}, t^{\prime}\right)=\left\langle\delta \mathbf{u}_{\perp}(\mathbf{r}, t) \cdot \delta \mathbf{u}_{\perp}\left(\mathbf{r}^{\prime}, t^{\prime}\right)\right\rangle,
$$

where

$$
\delta \mathbf{u}_{\perp}(\mathbf{r}, t)=\mathbf{u}_{\perp}(\mathbf{r}, t)-\left\langle\mathbf{u}_{\perp}(\mathbf{r}, t)\right\rangle
$$

is the fluctuation in the transverse-velocity component and the angular brackets denote an equilibrium average. If the simple fluid under consideration is also isotropic it will exhibit spatial and temporal invariance properties and $C\left(\mathbf{r}, t ; \mathbf{r}^{\prime}, t^{\prime}\right)$ may then be written as

$$
C\left(\mathbf{r}, t ; \mathbf{r}^{\prime}, t^{\prime}\right)=C\left(\left|\mathbf{r}-\mathbf{r}^{\prime}\right| ; t-t^{\prime}\right) .
$$

Moreover, for the purpose of general discussion and actual calculation it is convenient to use the spatial Fourier transform of $C(r, t)$, which is usually defined by ${ }^{11,12}$

$$
J_{\perp}(\mathbf{k}, t)=\int d \mathbf{r} C(r, t) e^{i \mathbf{k} \cdot \mathbf{r}}=\left\langle\delta \mathbf{u}_{\perp \mathbf{k}}^{*}(0) \cdot \delta \mathbf{u}_{\perp \mathbf{k}}(t)\right\rangle
$$

\section{A. The restricted case $\tau_{2}=0$}

The equation satisfied by $J_{\perp}(\mathbf{k}, t)$ may be obtained from the linearized equation of motion

$$
\rho \partial \mathbf{u} / \partial t=-\operatorname{div} \underline{\mathscr{S}}
$$

together with the linearized Eq. (2.12) and definition (3.4). This leads to

$$
\tau_{1} \partial^{2} J_{\perp} / \partial t^{2}+\left(1+l^{2} k^{2}\right) \partial J_{\perp} / \partial t+v k^{2} J_{\perp}=0,
$$

where $v=\eta_{s} \rho^{-1}$ is the kinematic viscosity and the parameters $\tau_{1}, l^{2}$ have been defined in Sec. II as

$$
\tau_{1}=-\alpha\left(T \mu_{10}\right)^{-1}, l^{2}=2 T \eta_{s} \beta^{2} / \mu_{20} \text {. }
$$

The initial value $J_{\perp}(\mathbf{k}, 0)$ is given by the equipartition theorem whereas $J_{\perp}^{\prime}(\mathbf{k}, 0)$, the initial condition for the time derivative of $J_{\perp}(\mathbf{k}, t)$, vanishes identically owing to the general time-reversal invariance properties of any equilibrium autocorrelation function. ${ }^{13}$ By taking the Laplace transform in time, Eq. (3.6) may be rewritten as

$$
\frac{\widetilde{J}_{\perp}(\mathbf{k}, s)}{J_{\perp}(\mathbf{k}, 0)}=\frac{s+\left(1+l^{2} k^{2}\right) \tau_{1}^{-1}}{s^{2}+\left(1+l^{2} k^{2}\right) \tau_{1}^{-1} s+v k^{2} \theta_{2}^{-1}}
$$

or taking the inverse transform

$$
J_{\perp}(\mathbf{k}, t)=J_{\perp}(\mathbf{k}, 0) e^{-a t / 2}\left[\cos (R t)+\frac{a}{2 R} \sin (R t)\right]
$$

with

$$
a=\left(1+l^{2} k^{2}\right) \tau_{1}^{-1}, \quad R^{2}=\frac{v k^{2}}{\tau_{1}}-\frac{a^{2}}{4} .
$$

The present analysis may be compared with that based on the microscopic Mori-Zwanzig theory ${ }^{11,14}$ which gives an exact equation for the time evolution of a correlation function in terms of a memory function, namely,

$$
\partial J_{\perp}(\mathbf{k}, t) / \partial t=-\int_{0}^{t} K\left(\mathbf{k}, t-t^{\prime}\right) J_{\perp}\left(\mathbf{k}, t^{\prime}\right) d t^{\prime} .
$$

It should be remarked that the memory kernel $K(\mathbf{k}, t)$ has a well-defined and unique expression in terms of projection operators. It can also be shown that $K(\mathbf{k}, t)$ may be written in the form

$$
K(\mathbf{k}, t)=k^{2} K^{\prime}(\mathbf{k}, t),
$$

which is usually down in generalized hydrodynamics. ${ }^{11,14}$ In this case the function $K^{\prime}(\mathbf{k}, t)$ is called the shear viscosity function or memory function for the transversevelocity correlation. Therefore, the generalized shear viscosity coefficient is identified with $\widetilde{K}^{\prime}(\mathbf{k}, s)$, where the tilde indicates the Laplace transform in time of $K^{\prime}(\mathbf{k}, t)$.

Usually the memory function is modeled mathematically. However, in our case this is unnecessary since by taking the Laplace transform in time of Eq. (3.9) and comparing with Eq. (3.8) we explicitly find that

$$
\widetilde{K}^{\prime}(\mathbf{k}, s)=\frac{v \tau_{1}^{-1}}{s+\left(1+l^{2} k^{2}\right) \tau_{1}^{-1}}
$$

or

$$
K(\mathbf{k}, t)=\frac{v k^{2}}{\tau_{1}} \exp \left[-\frac{1+l^{2} k^{2}}{\tau_{1}} t\right] .
$$

This equation shows that the relaxation time for the memory function

$$
\tau(k)=\tau_{1}\left(1+l^{2} k^{2}\right)^{-1}
$$

is $k$ dependent and is also related to the relaxation time $\tau_{1}$ for the stress tensor $\mathscr{\mathscr { S }}$. Incidentally, we remark that from Eq. (2.12) the relaxation equation for the fluctuations of $\mathscr{\mathcal { S }}$ in $k$ space is given by

$$
d \delta \underline{\mathscr{S}}_{\mathrm{k}} / d t=-\frac{1+l^{2} k^{2}}{\tau_{1}} \delta \underline{\mathscr{S}}_{\mathrm{k}}
$$

which shows that the relaxation time for the decay of these fluctuations is precisely the same as that of the memory function $K(\mathbf{k}, t)$.

The memory kernel $K^{\prime}(\mathbf{k}, t)$ must satisfy certain restrictions, the so-called sum rules. The second sum rule implies that $^{11}$

$$
K^{\prime}(\mathbf{k}, t=0)=\rho^{-1} G_{\infty}(k),
$$

where $G_{\infty}(k)$ is the high-frequency shear modulus. ${ }^{11,15}$ Also, consistency with hydrodynamics requires that

$$
\lim _{k \rightarrow 0} \lim _{s \rightarrow 0} \widetilde{K}^{\prime}(\mathbf{k}, s)=v \text {. }
$$

This last condition is obviously satisfied by Eq. (3.11) and implies that $v$ is $k$ independent. On the other hand, from Eqs. (3.12) and (3.15) we find

$$
\rho^{-1} G_{\infty}(k)=v \tau_{1}^{-1}
$$

and since by Eq. (3.7) $\tau_{1}=-2 \alpha \eta_{s}$ is independent of $k$, we conclude that in this case the high-frequency shear 
modulus must be $k$ independent as well. Furthermore, the ratio $\tau_{2}^{-1}=G_{\infty}(0) \eta_{s}^{-1}$ is identified with the Maxwell relaxation time of the usual viscoelastic theory. ${ }^{16}$

At this point it is worth emphasizing that the result expressed by Eq. (3.12) is precisely the exponential model which is introduced in generalized hydrodynamics for the memory function and which allows shear-wave propagation. ${ }^{11}$ However, we stress that in our development it arises naturally from the postulates of EIT and the only restrictions are $\tau_{2}=0$ and that the $\beta$ and $\mu_{10}$ phenomenological coefficients of Eq. (2.9) are spatially independent.

Recently Alley and Alder ${ }^{17}$ have studied, by computer simulations, the generalized shear viscosity coefficient $\eta_{s}(\mathbf{k}, s)$ in a fluid of hard spheres. They have shown that the zero-frequency and small- $k$ molecular-dynamics data may be fitted with an expression of the form

$$
\eta_{s}(\mathbf{k}, 0)=\left(1+a^{2} k^{2}\right)^{-1} \eta_{s}(0,0),
$$

where $a$ is an adjustable parameter with the dimensions of length. A physical interpretation for this parameter is obtained by considering a test particle of varying size immersed in the fluid. In this context, $a$ turns out to be a measure of the deviation from the Stokes friction law:

$$
F_{\text {drag }} / F_{\text {Stokes }} \cong 1-a \sigma_{12}^{-1} \text {, }
$$

where $\sigma_{12}=\frac{1}{2}\left(\sigma_{1}+\sigma_{2}\right)$ is the average diameter of the test particle and a bath particle. In this sense, $a$ incorporates the effects of spatial inhomogeneities into the hydrodynamical description.

Now, from Eqs. (3.10) and (3.11) it follows that

$$
\rho^{-1} \eta_{s}(\mathbf{k}, 0)=\lim _{s \rightarrow 0} k^{-2} \widetilde{K}(\mathbf{k}, s)=v\left(1+l^{2} k^{2}\right)^{-1}
$$

which has the same structure as Eq. (3.18). As mentioned in Sec. II, $l$ describes the range of the spatial inhomogeneities in the stress tensor $\mathscr{\mathscr { S }}$, and therefore has essentially the same physical interpretation as $a$. It should be emphasized that Eq. (3.20), which was obtained systematically from the postulates of EIT, is one of the central results of what is usually called generalized hydrodynamics. $^{11,17}$

\section{B. The general case}

We now consider the more general case in which $\tau_{2} \neq 0$ and where the relaxation of the system is described by the set of equations (2.11). By explicit algebraic manipulations one can eliminate $\mathscr{P}$ from Eqs. (2.11a) and (2.11b) and combine the resulting expression with Eq. (3.5). Introducing the transverse velocity $\mathbf{u}_{\perp}$ and making use of the definition (3.4) one arrives at the time evolution equation for $J_{\perp}(k, t)$, namely,

$$
\partial^{3} J_{\perp} / \partial t^{3}+A \partial^{2} J_{1} / \partial t^{2}+B \partial J_{\perp} / \partial t+C J_{\perp}=0
$$

where we have used the abbreviations

$$
\begin{aligned}
& A(k)=\left(\tau_{1}+\tau_{2}\right) / \tau_{1} \tau_{2}, \\
& B(k)=\left(1+l^{2} k^{2}-v \tau_{2} k^{2}\right) / \tau_{1} \tau_{2}, \\
& C(k)=v k^{2} / \tau_{1} \tau_{2} .
\end{aligned}
$$

Notice that if $\tau_{2}$ is set equal to zero in this expression, Eq. (3.21) reduces to (2.12). As mentioned earlier, it should be stressed that the initial conditions associated with Eq. (3.21) are not arbitrary. Rather, they are stipulated by the Mori-Zwanzig theory itself and the general time-reversal invariance properties that an arbitrary autocorrelation function should satisfy. ${ }^{13}$ One finds that $J_{\perp}(\mathbf{k}, 0)$ is determined from the equipartition theorem, that $J_{\perp}^{\prime}(\mathbf{k}, 0)=0$ and

$$
J_{\perp}^{\prime \prime}(\mathbf{k}, 0)=-K(\mathbf{k}, 0) J_{\perp}(\mathbf{k}, 0) .
$$

Following the same procedure leading to Eq. (3.8) and taking into account the above-mentioned initial conditions, we find that

$$
\widetilde{K}(\mathbf{k}, s)=-\frac{J_{\perp}^{\prime \prime}(\mathbf{k}, 0)}{J_{\perp}(\mathbf{k}, 0)} \frac{s-\left[J_{\perp}(\mathbf{k}, 0) / J_{\perp}^{\prime \prime}(\mathbf{k}, 0)\right] E k^{2}}{\left[s+\frac{1}{2} A(k)\right]^{2}+D^{2}(k)},
$$

where we have defined

$$
\begin{aligned}
& E=C(k) / k^{2}, \\
& D^{2}(k)=B(k)+\frac{J_{\perp}^{\prime \prime}(\mathbf{k}, 0)}{J_{\perp}(\mathbf{k}, 0)}-\frac{1}{4} A^{2}(k) .
\end{aligned}
$$

By taking the inverse Laplace transform of Eq. (3.24) we get

$$
\begin{aligned}
K(\mathbf{k}, t)=-\frac{J_{\perp}^{\prime \prime}(\mathbf{k}, 0)}{J_{\perp}(\mathbf{k}, 0)}[ & \cos D(k) t \\
- & \frac{1}{D(k)}\left[E k^{2} \frac{J_{\perp}(\mathbf{k}, 0)}{J_{\perp}^{\prime \prime}(\mathbf{k}, 0)}-\frac{1}{2} A(k)\right] \\
& \times \sin D(k) t] e^{-A(k) t / 2}
\end{aligned}
$$

Note that $K(\mathbf{k}, 0)=-J_{\perp}^{\prime \prime}(\mathbf{k}, 0) / J_{\perp}(\mathbf{k}, 0)$, which is precisely condition (3.23). Now, the second sum rule, Eq. (3.15), fixes the initial value $K(\mathbf{k}, 0)$ as

$$
K(\mathbf{k}), 0)=\left(k^{2} / \rho\right) G_{\infty}(k) .
$$

In contrast to what we have found in Sec. III A, the highfrequency shear modulus is now $k$ dependent. On the other hand, consistency with hydrodynamics is immediately verified since from Eqs. (3.16) and (3.24) it follows that

$$
v=1 / 2 T \rho \mu_{10}
$$

which is precisely the expression for $\mu_{10}$ obtained in Sec. II, namely, $\mu_{10}=\left(2 \eta_{s} T\right)^{-1}$.

\section{COMPARISON WITH MICROSCOPIC THEORIES}

There have been various attempts to derive the equations of generalized hydrodynamics from microscopic models using several of the well-known techniques of nonequilibrium statistical mechanics. In all of these attempts, the wave-vector- and frequency-dependent trans- 
port matrix has been formally expressed in terms of quantities depending on the dynamics of the underlying $\mathrm{N}$ body problem, thus making the explicit evaluation of its relevant elements considerably difficult. So far as we know, such a computation has never been carried out in terms of the molecular parameters and a given intermolecular potential for any system. The few calculations which have been performed to the stage where comparison with the experimental or computer results is feasible have relied upon the use of ad hoc models for the relevant dynamical quantities. Our main objective in this section will be to emphasize the fact that at least one of these models, the exponential memory function, which has been successfully used to account for the experimental data in argonlike fluids ${ }^{11}$ corresponds precisely to the one described by Eq. (3.12). In other less obvious cases, we shall content ourselves with a more qualitative comparison between the results of Sec. III A and those obtained from first principles.

The first efforts to derive frequency- and wavenumber-dependent transport coefficients with the idea of explaining the behavior of the correlation functions obtained by inelastic neutron scattering and computer simulations were based in linear-response theory. ${ }^{18,19}$ For the specific case of the time correlation function of the transverse velocity for a simple monatomic fluid, the formal expression generated by the theory was subsequently studied by a phenomenological approach selecting models that would satisfy all the pertinent sum rules and the lowfrequency and small-wave-number limit as required by classical linearized hydrodynamics. ${ }^{11,17}$ The results of this approach, both for the spectral density of the correlation function as well as for its form in $\mathbf{k}, t$ space, are identical in structure to Eqs. (3.8a) and (3.8b) consistent with the fact that an exponential function mimics the corresponding memory function [cf. Eqs. (3.8) and (3.3) of Ref. (18), and Eqs. (3.5.4) and (6.2.20) of Ref. (11)]. Since they have been used to explain the computer data for an argonlike fluid, we can use the corresponding numerical results to give estimates of our two undetermined parameters $\tau_{1}$ and l. In fact, comparison of Eq. (3.8a) of this paper and Eq. (3.8) of Ref. (18) leads to

$$
\Omega^{2}(k)=\left\langle\omega_{t}^{2}(k)\right\rangle-\frac{1}{4 \tau_{t}^{2}(k)}=\frac{k^{2} v}{\tau_{1}}-\frac{\left(1+l^{2} k^{2}\right)}{4 \tau_{1}^{2}}
$$

whence

$$
k^{-2}\left\langle\omega_{t}^{2}(k)\right\rangle=\rho^{-1} G_{\infty}(k)=v \tau_{1}^{-1}
$$

which is precisely Eq. (3.17). Furthermore,

$$
\frac{1}{\tau_{t}^{2}(k)}=\frac{\left(1+l^{2} k^{2}\right)^{2}}{\tau_{1}^{2}}=\frac{1}{\tau_{t}^{2}}+\left(k v_{0}\right)^{2},
$$

where $\tau_{t} \equiv \tau_{t}(0)=\tau_{1}=G_{\infty}^{-1} \eta_{s}$ is also consistent with our results. Ignoring the $k^{4}$ term in Eq. (4.3) we also see that

$$
2 l^{2} / \tau_{1}^{2}=2 k_{B} T / m=l^{2} / 4 \alpha^{2} \eta_{s},
$$

where use has been made of Eq. (3.7) so that

$$
\frac{l}{|\alpha|}=2\left(2 \eta_{s} k_{B} T / m\right)^{1 / 2} \simeq 70 \mathrm{~g} \mathrm{sec}^{-2}
$$

for argon at $85 \mathrm{~K}$. Independently, from Eq. (4.4) and using for $\tau_{1}$ the value of $0.26 \times 10^{-12} \mathrm{sec}$ (see Ref. 18) one finds that $|\alpha|=6 \times 10^{-11} \mathrm{~cm} \mathrm{sec}^{2} \mathrm{~g}^{-1}$ and thus $l \sim 4 \times 10^{-9} \mathrm{~cm}$. Thus, the correlation length of the spatial inhomogeneities in the stress tensor is of the same order of magnitude of the parameter $a$ in the Alley and Alder calculation ${ }^{17}$ for rigid spheres, where for $\sigma \sim 10^{-8}$ $\mathrm{cm}$ turns out to be of the order of magnitude of $10^{-9} \mathrm{~cm}$. These results may be considered as a strong support for the thermodynamical basis of generalized hydrodynamics, at least within the approximations of Sec. III A and for argonlike fluids.

The other calculations based upon linear-response theory ${ }^{19}$ lead to equations similar to Eq. (3.6) without the terms in the first time derivative of $J_{\perp}$ and with a more complicated $k$ dependence in the coefficient associated with $J_{1}$. Since the results are merely formal, no further comparison is feasible at this point.

Another microscopic model of special interest in dealing with frequency- and wave-number-dependent transport coefficients within a framework of ideas more closely related to EIT is the analysis of correlation functions based on the generalized Langevin-equation development of Mori and Zwanzig. ${ }^{20-22}$ Contrary to the calculations mentioned above, where only conserved variables are examined, the treatment includes also the dissipative fluxes as independent variables. In this way, a connection is established with EIT. Formal expressions for the spectral density of the transverse current correlations are obtained in which the influence of the fast variables is manifested, and essentially Eq. (3.6) is obtained with a factor $\omega_{s}(k)$ instead of $v k^{2}$ in the coefficient of $J_{1}$ [see Eq. (4.53a) of Ref. 20]. Yet in the comparison of the results for the case of the longitudinal current correlations with the computer data of Rahman some ad hoc simplifications are required. These lead to the Maxwell-Cattaneo-type equations for the fluxes, meaning that the essential part of the corresponding memory function will be an exponential, which once more is the essential result of our macroscopic analysis.

Finally, it is worth mentioning that Zubarev and Tischenko $^{23}$ have used a dynamic generalization of the Ornstein-Zernike theory to derive linearized hydrodynamical equations which are nonlocal in space and time. These authors start from the relaxation functions or transport kernels $\mathscr{L}_{m n}(\mathbf{r}, t)$ defined by

$$
\begin{aligned}
& \mathscr{L}_{m n}\left(\mathbf{r}-\mathbf{r}^{\prime}, t-t^{\prime}\right) \\
& =\Theta\left(t-t^{\prime}\right) e^{-\epsilon\left(t-t^{\prime}\right)}\left(J_{m}(\mathbf{r}, t), J_{n}\left(\mathbf{r}^{\prime}, t^{\prime}\right)\right),
\end{aligned}
$$

where $\Theta(t)=1$ for $t>0$ and $\Theta(t)=0$ for $t \leq 0$ and $\epsilon$ is a small parameter whose limit $\epsilon \rightarrow 0_{+}$is taken after the thermodynamic limit has been performed. Here,

$$
\begin{aligned}
\left(J_{m}(r, t), J_{n}\left(r^{\prime}, t^{\prime}\right)\right) & \\
\quad= & \int_{0}^{1} d \tau\left\langle J_{m}(\mathbf{r}, t)\left[\rho_{0}^{\tau} J_{n}\left(\mathbf{r}^{\prime}, t^{\prime}\right) \rho_{0}^{-\tau}-\left\langle J_{n}\left(r^{\prime}\right)\right\rangle\right]\right\rangle,
\end{aligned}
$$

where $J_{0}$ denotes heat flux, $J_{1}$ momentum flux, and $\rho_{0}$ the grand canonical function. Due to space isotropy, the Fourier transforms of the kernels $\mathscr{L}_{m n}$ can be represented as 


$$
\begin{aligned}
& \mathscr{L}_{00}(\mathbf{k}, \omega)=k^{2} L_{00}(k, \omega) \\
& \mathscr{L}_{01}^{\alpha}(\mathbf{k}, \omega)=i k_{\alpha} L_{01}(k, \omega), \\
& \mathscr{L}_{10}^{\alpha}=i k_{\alpha} L_{10}(k ; \omega), \\
& \mathscr{L}_{11}^{\alpha \beta}(\mathbf{k}, \omega)=k_{\alpha} k_{\beta} L_{11}(k, \omega)+\left(\delta_{\alpha \beta} k^{2} k_{\alpha} k_{\beta}\right) L_{22}(k, \omega),
\end{aligned}
$$

where $L_{n n}(k, \omega)$ are scalar functions depending only on the magnitude of $k$ but not on its direction.

In order to be consistent with Fourier and NewtonStokes laws, one obtains in the low-frequency, longwavelength limit,

$$
\begin{aligned}
& L_{00}(k, \omega)=\lambda, \quad L_{01}(k, \omega)=L_{10}(k, \omega)=0, \\
& L_{11}(k, \omega)=\left(\zeta+\frac{4}{3} \eta_{s}\right), \quad L_{22}(k, \omega)=\eta_{s}
\end{aligned}
$$

with $\lambda, \zeta$, and $\eta_{s}$ being, respectively, thermal conductivity, bulk viscosity, and shear viscosity. The relaxation time $\tau$ and the correlation length $l$ may be written then in terms of the moments of the correlation functions. Indeed, if one defines

$$
L_{n n}\{q, s\}=\int d t \int d \mathbf{r}|\mathbf{r}|^{q} t^{s} L_{n n}(\mathbf{r}, t)
$$

one obtains

$$
\begin{aligned}
& L_{n n}=L_{n n}\{0,0\}, \\
& l^{2}=L_{22}\{2,0\} / 6 L_{22}\{0,0\}, \\
& \tau_{1}=L_{22}\{0,1\} / L_{22}\{0,0\} .
\end{aligned}
$$

These expressions given the transport coefficients, the relaxation times, and the correlation lengths in terms of the moments of the correlation functions, which are the quantities usually studied in microscopic theories. Equations (4.9) are the usual Kubo formulas, and the expressions for $\tau_{1}$ and $l$ constitute a generalization of those formulas to second-order coefficients, some of which have been used in this paper.

When localized about a fixed point in space, Zubarev and Tischenko's equations lead to differential equations for the conserved variables which are of the hyperbolic kind, thus implying that a Maxwell-Cattaneo equation of the linear type for a nonconserved variable, Eq. (2.10c), has been used in its derivation. The paper does show that in some special limits their formalism is adequate to treat effects such as the anomalous skin effect in solids.

\section{CONCLUDING REMARKS}

Several of the points raised in this paper deserve some additional comments stressing their physical content. The linearized equations of motion for the state variables obtained in Sec. III were shown to give rise in a particular case to the results of generalized hydrodynamics as it is handled at present, consistent with experimental and computer results.

For the more general case dealt with superficially in Sec. III B, we are still unable to evaluate its full physical meaning. In general, linear differential equations of the type handled here will give rise to exponential or modulated exponential memory functions. In the case of Eq. (3.21) we know that it will yield a sum of exponentials but their detailed form and the underlying hydrodynamics are still far from being clear. Hopefully their clarification will be able to cast some light on the nature of linear non-Markovian processes.

It should also be emphasized that the above comments apply to the case studied here, transverse-velocity correlations. Although there are strong indications that they will also be valid in other similar problems such as longitudinal velocity correlations, self-diffusion, and others, these systems wait to be solved explicitly.

In any case, the microscopic theories that have been developed have the necessary ingredients to provide for the foundations of generalized hydrodynamics, but so far no method has explicitly shown how to compute frequency- and wave-number-dependent transport coefficients. Extended irreversible thermodynamics, with all its limitations, seems to provide a good method for establishing the basis behind the use of explicit memory functions in performing such computations.

Finally, it is worth stressing that the more interesting case of longitudinal velocity correlations, which would allow comparison with neutron scattering and other real experimental results, and nonlinear cases are still to be considered. Yet, the ensuing mathematical difficulties are not easy to surmount, but we hope that something can be said about their inclusion in the near future.

\section{ACKNOWLEDGMENTS}

We gratefully acknowledge the financial support of the Programa de Cooperación Internacional con Iberoamérica y Filipinas of the Spanish Ministry of Educación y Ciencia, and of our universities (Universidad Nacional Autonoma de México and Universitat Autónoma de Barcelona). One of us (M.L.H.) also acknowledges to Consejo Nacional de Ciencia y Tecnologia (CONACYT) of Mexico for support in his visit to Spain. The partial support of the Comisión Asesora de Investigación Científica $y$ Técnica (CAICYT) of the Spanish Government is also acknowledged.
*Also at El Colegio Nacional, México, Mexico.

${ }^{1}$ R. F. Rodríguez, L. S. García-Colin, M. López de Haro, D. Jou, and C. Pérez-Garcia Phys. Lett. A 107, 17 (1985).

${ }^{2}$ G. Lebon, D. Jou, and J. Casas-Vázquez, J. Phys. A 13, 275 (1980).

${ }^{3}$ Recent Developments in Nonequilibrium Thermodynamics, Vol. 199 of Springer Lecture Notes in Physics, edited by J. Casas-
Vázquez, D. Jou, and G. Lebon (Springer, Berlin, 1984).

${ }^{4}$ D. Jou and C. Pérez-García, Physica (Utrecht) 104A, 320 (1980); C. Pérez-García, in Ref. 3.

${ }^{5}$ D. Jou, F. Bampi, and M. Morro, J. Non-Equilib. Thermodyn. 7, 201 (1982).

6J. E. Llebot, D. Jou, and J. Casas-Vázquez, Physica (Utrecht' 121A, 552 (1983). 
${ }^{7}$ F. Bampi, A. Morro, and D. Jou, Physica (Utrecht) 107A, 393 (1981).

${ }^{8}$ S. Hess, Z. Naturforsch. 32A, 678 (1977).

${ }^{9}$ L. Garcia-Colin and G. Fuentes, J. Stat. Phys. 29, 387 (1982).

${ }^{10}$ C. Pérez-García and D. Jou Phys. Lett. A (to be published).

${ }^{11}$ J. P. Boon and S. Yip, Molecular Hydrodynamics (McGrawHill, New York, 1980), Chap. 2.

${ }_{12}$ R. Mountain, Adv. Mol. Relaxation Processes 9, 225 (1977).

${ }^{13} \mathrm{H}$. Ted Davids, in Advances in Chemical Physics, edited by $\mathrm{I}$. Prigogine and S. Rice (Wiley-Interscience, New York, 1973), Vol. 24, p. 257.

${ }^{14}$ B. J. Berne and R. Pecora, Dynamic Light Scattering with Applications to Chemistry, Biology and Physics (Wiley, New
York, 1976), Chap. 11.

${ }^{15}$ R. Zwanzig and R. Mountain, J. Chem. Phys. 43, 4464 (1965). 16J. Frenkel, Kinetic Theory of Liquids (Dover, New York, 1955).

${ }^{17}$ W. E. Alley and B. J. Alder, Phys. Rev. A 27, 3158 (1983).

${ }^{18}$ C. H. Chung and S. Yip, Phys. Rev. 182, 323 (1969).

${ }^{19}$ Ph.A. Selwyn and I. Oppenheim, Physica (Utrecht) 54, 161 (1971).

${ }^{20}$ A. Z. Akcasu and E. Daniels, Phys. Rev. A 2, 962 (1970).

${ }^{21}$ M. Grant and R. Desai, Phys. Rev. A 2, 962 (1970).

22R. Bansal, Phys. Rev. A 16, 2191 (1977).

${ }^{23}$ D. N. Zubarev and S. V. Tischenko, Physica (Utrecht) 59, 282 (1972). 